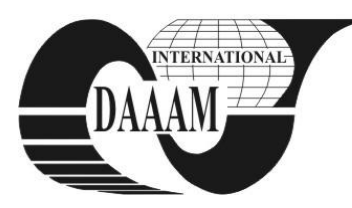

\title{
A NOVEL APPROACH TO MEASURE MACHINE RECONFIGURABILITY IN RECONFIGURABLE MANUFACTURING SYSTEM
}

\author{
GOYAL, K[apil] K[umar]; JAIN, P[ramod] K[umar] \& JAIN, M[adhu]
}

\begin{abstract}
The reconfigurable manufacturing system is a new class of manufacturing system which offers the capacity and functionality that is needed and when it is needed by adjusting its modular structure. Reconfigurability is achieved through changing the hardware and software modules in the modular reconfigurable machine tools. In the present work, authors propose a systematic approach to measure the reconfigurability at machine level which will help in measuring the responsiveness offered by the machines. The developed approach is illustrated through suitable example.
\end{abstract}

Key words: reconfigurable manufacturing system, reconfigurability, reconfigurable machine tool

\section{Notations:}

$M C_{i}^{j} \quad$ machine $i(1<i<I)$ in its $j^{\text {th }}\left(1<j<J_{i}\right)$ configuration $J_{i} \quad$ number of configurations offered by machine $i$

$A M_{i, j} \quad$ a set of auxiliary modules required in machine $i$ with its $j^{\text {th }}$ configuration

$M C_{p}^{q}$ machine $p$ with configuration $q$ for which reconfigurability is to be measured

\section{INTRODUCTION}

Manufacturing in the present scenario, is undergoing a shift from mass production to mass customization. Such a shift requires highly responsive manufacturing systems. The reconfigurable manufacturing system (RMS) justifies the need of hour by combining the high throughput of dedicated manufacturing system with the flexibility of flexible manufacturing systems (Koren et al., 1999). The most significant feature of the RMS is that the configuration of these systems evolves over a period of time in order to provide the functionality along with the capacity needed, and when it is needed (Mehrabi et al., 2000). The most significant ingradient of the reconfigurable manufacturing systems is the reconfigurable machine tool (RMT), which plays a vital role in

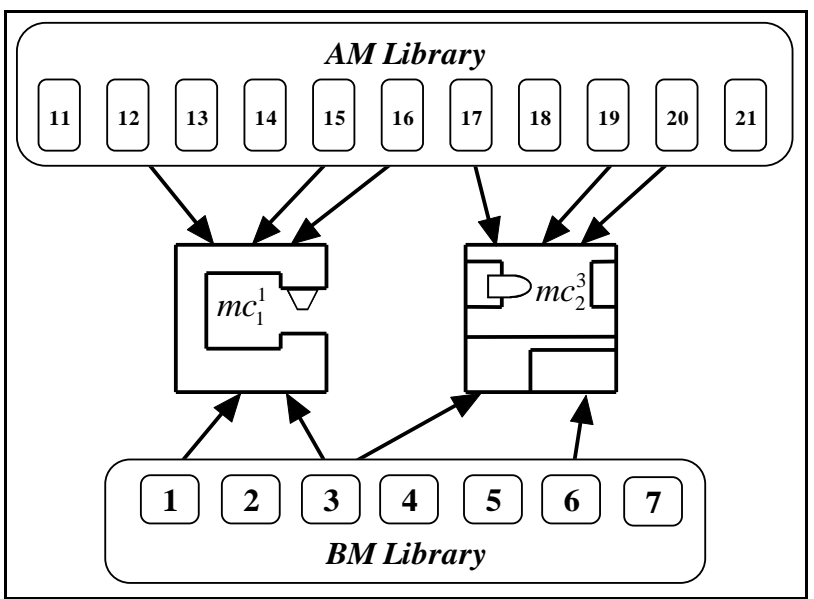

Fig. 1. Configuring RMT through module library adapted from (Pattanaik L.N., et al., 2007) achieving the adaptability and responsiveness through changes in its moduler structure. The RMTs are composed of basic modules and auxiliary modules. The basic modules are structural in nature like base, columns, and slide ways and the auxiliary modules are kinematical or motion providing modules such as spindle heads, tool changers, spacers, indexing units, adapter plates and angle structure, etc. As depicted in Fig. 1. the RMTs are configured using module library cantaining the commercially available basic modules and auxiliary modules. An RMT offers many configurations by keeping the basic modules and adding/removing and/or readjusting the auxiliary modules e.g. as given in Tab. 1 , the machine $M_{l}$ offers in total four configurations. Thus each machine configuration of machine 1 can be further converted into another three configurations with each having different configuration efforts depending on auxiliary module interactions. In the present paper authors propose a novel approach for measuring the reconfigurability of an RMT based on number of configurations into which a machine configuration can be converted and the reconfiguration effort required in all such conversions.

\section{LITERATURE REVIEW}

Maier-Speredelozzi et al. (2003) defined the system convertibility as the capability of a system to adjust production functionality and presented the system convertibility metrics based on the assessment of convertibility of the system configuration, machines and material handling equipments. Hence through such metrics it is very difficult to precisely grade the reconfigurability of a machine. The reconfigurability of the RMS on system level has been suggested based on the core characteristics of the system like modularity, scalability, convertibility and diagnosability (Gumasta et al. 2011). The developed index does not consider the module interactions at the machine level reconfigurations, which is the key enabler of the reconfiguration.

Thus considering the importance of reconfigurability in the RMS and lack of literature on machine level reconfigurability, in the present work a comprehensive approach to measure the reconfigurability on machine level has been proposed.

\section{MACHINE RECONFIGURABILITY}

The quick adaptability of the reconfigurable manufacturing system in response to the dynamic environment is achieved by reconfiguration of the machines. Thus reconfigurability is a criterion to judge the adaptability of the machine configuration. In the present work a novel approach to measure the reconfigurability of an RMT is proposed based on the number of configurations into which an existing machine configuration may be converted along with considering the effort required in conversions in the form of adding/removing and/or readjusting the auxiliary modules. The effort in each configuration conversion is being calculated by a methodology based on set theory. As shown in Fig. 2 in each conversion two 


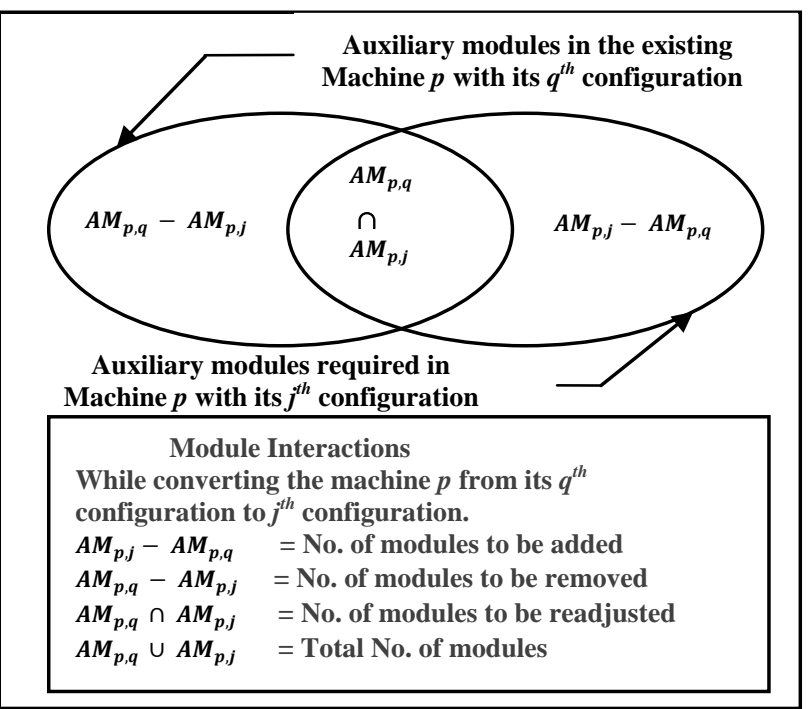

Fig. 2. Module interactions during configuration conversion

sets of auxiliary modules are participating one is the set of auxiliary modules of existing machine configuration and the other is the set of auxiliary modules required in the new configuration. Thus the total auxiliary modules i.e. union of the both sets of auxiliary modules is categorized into three classes, the auxiliary modules to be added, removed and readjusted. Here it is assumed that the existing modules which are retained in the next configuration need to be readjusted. Further the ratio of three classes of auxiliary modules to the total modules is multiplied by the weights $\alpha, \beta$ and $\gamma$ which gives the effort required in machine configuration conversion. In this way the total effort required for all the possible conversions of existing configuration is calculated.

For computing the reconfigurability of a machine configuration, the number of configurations into which it can be converted plays a vital role, if a machine is having only one configuration $\left(J_{p}=1\right)$ i.e. it cannot be converted into further any configurations, thus it will not make any contribution in reconfigurability. As the number of configurations into which a machine can be converted increases, its contribution to the reconfigurability also increases. As the objective in general is to maximize the reconfigurability, therefore every additional increase in the number of possible configurations must reflect an increased value of reconfigurability. Therefore the reconfigurability contribution of every additional increase in the $J_{p}$ must reflect more pronounced value of reconfigurability as compared to that of $J_{p}-1$. To reflect this consideration a power index $\mathrm{z}$ is used in the equation (1). Therefore the reconfigurability $\left(R_{p, q}\right)$ of a machine configuration is calculated using the following equation.

$$
R_{p, q}=\frac{\left[J_{p}-1\right]^{Z}}{\sum_{j=1, j \neq q}^{J p}\left[\alpha \frac{A M_{p, j}-A M_{p, q}}{A M_{p, q} \cup A M_{p, j}}+\beta \frac{A M_{p, q}-A M_{p, j}}{A M_{p, q} \cup A M_{p, j}}+\gamma \frac{A M_{p, q} \cap A M_{p, j}}{A M_{p, q} \cup A M_{p, j}}\right]}
$$

Generally $\alpha>\beta>\gamma$, as the effort required in adding the module is comparatively higher than removing the module and further the effort required in removing the module is reasonably higher than just readjusting the existing modules.

\section{ILLUSTRATION}

In the present study the values of $\alpha, \beta, \gamma$ are taken as 0.5 , $0.4,0.1$ respectively and the value of $\mathrm{z}$ is assumed as 2 . The reconfigurability of the machine configuration $M C_{2}^{3}(p=2, q=3)$ in the Tab. 1 is computed for illustration. The number of possible configuration $J_{p}$ into which $M_{2}$ can be converted is 5, thus the machine $M C_{2}^{3}$ can be further converted into 4

\begin{tabular}{|c|c|c|c|c|}
\hline & Configuration & Modules & & Reconfigurability \\
\hline \multirow{4}{*}{$M_{1}$} & $M C_{1}^{1}$ & $\{1,5\}$ & $\{13,17,21,22\}$ & 8.20 \\
\cline { 2 - 5 } & $M C_{1}^{2}$ & $\{1,5\}$ & $\{12,13,15,20,21\}$ & 8.25 \\
\cline { 2 - 5 } & $M C_{1}^{3}$ & $\{1,5\}$ & $\{1,17,18,20,21\}$ & 8.80 \\
\cline { 2 - 5 } & $M C_{1}^{4}$ & $\{1,5\}$ & $\{15,17,18\}$ & 7.74 \\
\hline \multirow{4}{*}{$M_{2}$} & $M C_{2}^{1}$ & $\{2,4,8\}$ & $\{11,13,16,22,24\}$ & 11.52 \\
\cline { 2 - 5 } & $M C_{2}^{2}$ & $\{2,4,8\}$ & $\{14,16,19\}$ & 9.77 \\
\cline { 2 - 5 } & $M C_{2}^{3}$ & $\{2,4,8\}$ & $\{13,19,24\}$ & 10.45 \\
\cline { 2 - 5 } & $M C_{2}^{4}$ & $\{2,4,8\}$ & $\{11,13,15,18,24\}$ & 11.67 \\
\cline { 2 - 5 } & $M C_{2}^{5}$ & $\{2,4,8\}$ & $\{11,14,18\}$ & 10.03 \\
\hline
\end{tabular}

Tab. 1. Reconfigurable machine tool configuration details

configurations i.e. $\left[J_{p}-1\right]$ is $[5-1]=4$ in this case. The effort in each of these conversions is computed and summed up in the denominator in equation (1). We illustrate the effort involved in the conversion of present machine configuration $M C_{2}^{3}$ into $M C_{2}^{1}$. During this conversion the number of modules to be added $\left(A M_{p, 1}-A M_{p, q}\right)$ are $3\{11,16,22\}$, number of modules to be removed $\left(A M_{p, q}-A M_{p, 1}\right)$ is $1\{19\}$ and number of modules to be readjusted $\left(A M_{p, q} \cap A M_{p, 1}\right)$ are $2\{13,24\}$. The total number of modules $\left(A M_{p, q} \cup A M_{p, 1}\right)$ in this conversion are $6\{11,13,16,19$, $22,24\}$. Thus effort required in this conversion is computed as $(0.5 * 3 / 6+0.4 * 1 / 6+0.1 * 2 / 6)$ which is 0.35 . Similarly the effort required in other three conversions $\left(M C_{2}^{2}, M C_{2}^{4}, M C_{2}^{5}\right)$ is $0.38,0.35$, 0.45 . Thus total effort involved in all the four conversions (denominator of equation (1)) is 1.53 and the machine reconfigurability $\left(R_{p, q}\right)$ of $M C_{2}^{3}$ is $\left((5-1)^{2} / 1.53\right)=10.45$. The values of machine reconfigurability are updated in the Tab. 1.

\section{CONCLUSION AND FUTURE SCOPE}

In the present work a comprehensive approach has been developed to measure the reconfigurability of a machine tool based on the number of configurations into which a machine can be converted and the total effort required in such conversions. The development of this performance parameter for machines will be of great help in the machine selection problem in an environment, where a large number of machines are available to perform an operation. Thus along with the cost the reconfigurability can also be considered as a selection criterion which will indicate the level of responsiveness for the machine in consideration. The proposed methodology can easily be extended to measure the reconfigurability of the manufacturing lines/cells. In future authors plan to apply the developed approach on the selection of machines for a reconfigurable manufacturing environment.

\section{REFERENCES}

Gumasta, K.; Gupta, S.K.; Benyoucef, L. \& Tiwari, M. K. (2011). Developing a reconfigurability index using multiattribute utility theory. International Journal of Production Research, 49: 6, pp. $1669-1683$

Koren, Y.; Hiesel, U.; Jovane, F.; Moriwaki, T.; Pritschow, G.; Ulsoy, G. \& Van, B.H. (1999). Reconfigurable Manufacturing Systems, Annals of the CIRP, 48(2), pp. 527-540

Maier-Speredelozzi, V.; Koren, Y. \& Hu, S.J. (2003). Convertibility measures for manufacturing systems. Annals of the CIRP, 2003, 52, pp. 367-370

Mehrabi, M.G.; Ulsoy, A.G. \& Koren, Y. (2000). Reconfigurable manufacturing systems: Key to future manufacturing. Journal of Intelligent Manufacturing, 11(4), pp. 403-419

Pattanaik L.N.; Jain P.K. \& Mehta N.K. (2007). Cell formation in the presence of reconfigurable machines, International Journal of Advanced Manufacturing Technology, 34, pp. 335-345

\begin{tabular}{|l|l|l|l|l|}
\hline Machine & Machine & Basic & Auxiliary Modules & Machine \\
\hline
\end{tabular}

